\title{
Easing Cross-Border Communication: MOBILE-mediated Communication and Its Framework
}

\author{
Kyungsub Stephen Choi \\ School of Management \\ Rhode Island College \\ Providence, RI, USA 02908 \\ kchoi@ric.edu
}

\author{
Youngsoo Kim \\ School of Information Systems \\ Singapore Management University \\ Singapore 178902 \\ yskim@smu.edu.sg
}

\begin{abstract}
Communication is probably the most critical component of an organization engaged in a crossborder collaboration. Today's smart devices substantially contribute to such communication. Combined with social media, mobile communication technologies are becoming the main platform for many core functions within organizations. In this paper, we identified seven media identifiable attributes: synchronicity (SYN), de-individuation and co-presence $(D C P)$, accessibility readiness (ARD), cognizance of environment change (CEC), wearability-portability (WRB) modality-select (MDS) and visibility (VSB). These seven attributes significantly impact the course of mobile-mediated communication. We believe that development of a theoretical perspective that embraces the complexity of mobile-mediated communication is due in order to fully comprehend the mobile ecosystem that is upon us.
\end{abstract}

\section{Introduction}

Beginning with iPhone and Android phones about a decade ago, mobile technologies have brought significant change to both the business and personal lifestyle landscape. In order to keep up with this rapidly evolving environment, many business organizations are hastily mobilizing their customerfocused operations [25], [33].

In the case of the consumer market, industry numbers and figures report that the consumption rate of mobile technologies is rapidly increasing. One industry report states that worldwide smartphone sales reached 1.2 billion units in 2014, up 28.4 percent from 2013, representing two-thirds of global mobile phone sales [16]. Similarly, the mobile operating system (OS) market is also exhibiting fast growth, with the Android OS topping all other mobile operating systems. Even the leading desktop OS, the Windows OS, has become much more mobile friendly [22]. In contrast, personal computer software sales and demand are experiencing a significant decline [21], [27].

Mobile technology has had a similar impact on the social media sector. The two leading social media platforms, Facebook and Twitter, both reported that the overwhelming majority of their users are mobile users; $80 \%$ of Twitter active users are on mobile [31] and $85 \%$ of Facebook daily active users are on mobile [12]. An industry report predicts that "Facebook is now so thoroughly a mobile service that its original website may soon become a footnote in the company's financial statements" [17]. Accessing social media such as Facebook or Twitter through smartphones or mobile devices is becoming the preferred choice of many people today. This is yet another example of the way mobile technology is significantly influencing consumer behavior and lifestyles, as well as the overall business environment.

The significance of this phenomenon is that communication through this combination (mobile device and social media) is drastically different from the communication that occurs when either one is used alone or in combination with only a non-mobile or less-mobile setup like a PC [7]. Further magnifying this effect is wearable technology (e.g. Google Glass and the Apple Watch). This technology possesses physical characteristics that influence how a user initiates and conducts communication and information sharing. One such characteristic is 'wearability.' This refers to how well and naturally a device can be worn and used. Moreover, if a device is too visible or too easily discerned by others during communication, then it may negatively influence a conversation or even cause non-users to completely shun the user, as was the case with Google Glass [3]. This characteristic has emerged as a major technical challenge and a factor that impacts whether the wearable device becomes popular.

Under these circumstances, from the academic perspective, it is worth initiating more IT-enabled 
communication studies whose aim should be to explore a number of issues stemming from the ever-increasing prevalence and significance of the mobile platform. To ensure the efficacy and rigorousness of these studies, a sound media theory is imperative. Of course, the information systems community has a number of such theories (e.g. Media Synchronicity Theory [10] that have sustained computer-mediated communication (CMC) studies. However, the issue now is that media synchronicity theory (MST) and other established theories are not adequate for addressing and explaining the peculiarities (e.g. wearability) of mobile technologies and the myriad issues that arise from them. Given the expectation that there will be increasingly more mobile communication devices in various forms in coming years, it is important that we as an academic community develop an appropriate theoretical framework.

This is an on-going report on a study that seeks to develop a theoretical framework that can eventually yield a sound media theory for IS studies. In this report, the following sections are provided: 1) relevant CMC theories, 2) media characteristics and capabilities, 3) research issues, and 4) expected contribution.

\section{Relevant computer-mediated communication theories}

Among CMC theories, the two most well-known ones are Media Richness Theory (MRT) and Media Synchronicity Theory (MST). For this preliminary paper, the theories' brief descriptions are provided here.

MRT is based on two criteria: uncertainty and equivocality under an organizational setting [8]. Uncertainty is defined as the gap between what information is needed and what information is already available. Equivocality occurs when c multiple, conflicting interpretations are present or when no one particular interpretation stands out. A medium is evaluated according to how well it integrates these two criteria following a scale of high and low. Thus, a medium with high media richness is one that exhibits both low uncertainty and low equivocality. For example, face-to-face communication is a medium that shows high media richness according to MRT, while an unaddressed document is a medium that shows low media richness.

Media Synchronicity Theory (MST) [10] divides media capabilities into the following categories: transmission velocity, parallelism, symbol sets, rehearsability and reprocessability. Transmission velocity refers to the speed by which the medium conveys its messages. Parallelism concerns the medium's capability to simultaneously send multiple messages to different recipients. Symbol sets are the number of physical expressions that one can add to a message, such as a handshake, the nodding of the head or the closing of the eyes. Rehearsability is a measure of the degree to which the medium allows a message to be edited and rehearsed before sending. Similarly, reprocessability describes the degree to which a medium allows a message to be re-evaluated again during decoding.

\section{Media characteristics and capabilities of mobile-mediated communication}

The two theories described above have provided the theoretical framework for $\mathrm{CMC}$ studies for decades. But as wearable mobile ICT devices increasingly become more common, these frameworks fall short in addressing the new behaviors and perceptions that are caused by the devices' physical attributes.

On the other hand, in Mobile-mediated Communication (MMC) theory, the focus is centered on mobility, mobile-related functions and behaviors, and device wearability. Earlier, during the cellular phone era, Castells et al [6] studied many of the social dimensions of mobile communications use in our society. In their work, they describe the significant attributes of mobile platform communication. However, their research was primarily focused on voice communication which does not include the multimedia and data sharing that is so prevalent now.

Thus, when we see that the breadth of today's media is wider and more complex, the limitations of previous frameworks becomes more evident. Simply put, there are now many more kinds of multimedia and mobile applications. Today, through the Apple store or Android market, one can browse hundreds of different types of mobile applications across a variety of genres. Similarly, there exist a number of different social platforms tailored to suit different individuals and lifestyles.

Similarly, the mobile device market continues to surprise the world with creative products such as Google Glass and the Apple Watch. Given the increasingly ready availability of Nano technology [24] and the increasing invention of smaller personal gadgetry, it would not be a surprise to see such items as a necklace, ring, wrist band or contact lenses become mobile devices in the near future.

The attributes and features of these various multimedia and mobile applications, as well as their integration with each other, culminates in the creation 
of new levels and paradigms of communication. Furthermore, these paradigms have opened new areas of and opportunities for value creation.

One such new attribute is visibility (or more specifically, the lack thereof). In order for wearable mobile devices to be successful, one industry report highlights the importance of a device's ability to be hidden [3]. In other words, can the device be worn by a person, yet hide itself from other people by its design or size in order to minimize the attention it draws? This attribute is one of several that helps predict how people will behave when using these wearable devices. Along with visual prominence, attributes such as design, size and wearability will become significant factors in one's decision whether to use these devices for communication purposes.

MMC is a theory which will address the attributes, needs and limitations described above, which is why we believe it is better situated to accommodate the ever-changing nature of mobile communication platforms, and will provide a theoretical framework for scholarly work on these mobile devices.

The first step in this endeavor is to define the prominent media capabilities that differentiate one media from another. The information filtering capability has been excluded as it is one of the common features in almost all ICT and does not differentiate one media from another media. Additionally, due diligence was exercised in excluding any no-value-added capabilities. For example, any capability that differentiate only between face-to-face to a media was excluded since face-to-face is not in the scope of MMC.

Therefore, the major capabilities to be studied are: synchronicity (SYN), de-individuation and co-presence (DCP), accessibility readiness (ARD), cognizance of environment change (CEC), wearability-portability (WRB) modality-select (MDS) and visibility (VSB).

Synchronicity (SYN) is a core communication process of mobile technology. The media synchronicity theory [10] used this to sub-categorize the processing and transmission capabilities. Given the robust wireless connectivity available to many mobile users, flawless and simultaneous communication can surely expected between two or more parties in various communication modes (voice, texting, or video).

De-individuation and co-presence (DCP) occurs when a person simply observes an on-going group communication without taking a part in that communication (de-individuation), which in turn may allow the person to be involved in more than one online rendezvous (co-presence). In other words, a person may be in and out of a few different online meetings simultaneously. Previous studies [20], [13] hint that these behaviors are common in group contexts and more so in a technology-driven setting. If an online meeting is text-driven then these behaviors are more profound. By the expectancy-value theory [32], a person is perceived to gain more goods by engaging in multiple conversations and acquiring more information. Additionally, this is highly correlated to the synchronicity capability of a mobile technology. Without a robust synchronous maneuver, these behaviors are not possible.

Cognizance of environment change (CEC) is one of the prime functions of mobile technology - the ability to react to changing locations [18]. Location-based data and information is a major function of mobile technology and it has sparked many academic research studies [5] and commercial applications. For example, the "Foursquare" mobile application gathers information about businesses and places around a person's location [26]. Based on the ratings and comments of the previous visitors, a person may evaluate a certain nearby business establishment before patronizing it. Another example is that of "Zalando" [19], an image recognition mobile application that enables a user to take image shots of people passing by in order to get information (such as brand names) on what they are wearing and where to purchase similar clothes.

Modality-select (MDS) describes a person's freedom to choose or switch back and forth from one communication mode to another. For example, a person sending a message may choose to make a voice call, send a text message either by phone or through a social networking medium, or begin video conferencing. In return, the receiver may respond back through the same communication mode or choose to use other channels. For example, many parents like to call their children in order to hear their children's voices, but the children like to text instead of voice call [9], [23].

Accessibility readiness (ARD) measures the level of readiness of a mobile technology for communication. Readiness is determined by the specific maneuvers that a person needs to take in order to use the mobile technology for communication, beginning with the location where the technology initially resides in normal use and ending at the point where a person successfully forwards a message to another person. The level of readiness varies with technology: in the case of a personal computer, there are many maneuvers required until a person can respond to an email message, but a "thumb drive" smartphone takes less number of maneuvers.

An example of technology with particularly high readiness is the "pull" technology utilized in such devices as Google Glass. It is a technology where a person or object is identified via image 
reading/recognition software, which then "pulls up" any associated data and information automatically, without a person's conscious intervention or physical steps. This pull technology is based on image-reading technology which is actively applied in mobile applications [15], [19].

Wearability-portability (WRB) refers to a how well an item of mobile technology conforms to a person's physical needs (e.g. "how well does a device fit or how wearable it is for a person engaged in on-demand communication"). It has to fit to a person where he can function his or her daily routines without causing them to be conscious of the fitness of the mobile technology to their body.

Early in the development of mobile technology, the paradigm was portability: a device had to be portable for a person to carry it. Today, this paradigm is rapidly shifting to wearability. While the mobile communications field is still dominated by portable smartphones and tablet PCs (e.g. the iPad), we are witnessing a major shift to wearable smart devices. The underlying idea that drives this paradigm has much in common with embedded computing [4], [28]. Here, communication is initiated as naturally and effortlessly as possible if a device is not only portable, but also wearable.

Finally, Visibility (VSB) refers to the degree to which a piece of mobile technology's outside appearance draws the attention of other people. In other words, the question becomes how visible (or how small) the device is and how it is perceived by others. The common consensus is that the smaller, more hidden and more intuitive a device is, the more likely it is to be accepted by other people. Ignoring this fact has led to noticeable failures [3]. Therefore, the growing expectation of people who are consuming these technologies is that the smaller the device, the more naturalness it possesses. This paradigm explains the ever-decreasing size of emerging wearable mobile devices.

\section{Media capabilities classification}

The media capabilities described in the previous section can be placed into two groups. One group is oriented toward the communication process, while the other group is more closely associated with outside physical appearance. The capabilities associated with the communication process are synchronicity (SYN), de-individuation and co-presence (DCP), cognizance of environment change (CEC), while the ones associated with the physical appearance are modalityselection (MDS), accessibility readiness (ARD), wearability-portability (WRB) and visibility (VSB).
The communication process group encompasses those capabilities that are integrated with social media and smartphones, categorized by their outstanding communication features. Among these capabilities, the most significant is that of cognizance of environment change (CEC). This capability yields new data and information based on changes in location and time. Such information can also be acquired automatically via "pull" technology. Given these available technologies-image-reading and the automatic "pulling" of data and information - a person can load himself with endless streams of data, information and communication.

It is important to note that these capabilities - CEC, SYN, and DCP - interact dynamically and reciprocally with each other in a group setting. For instance, synchronicity (SYN) closely sustains de-individuation and co-presence (DCP). While a person is exercising DCP, he must be synchronously connected and informed on the on-going activities. This ensures that the person is able to continue his or her participation in the group action and process.

The synchronicity (SYN) capability also allows cognizance of environment change (CEC) to be effective. A device's continuous ability to track location-change and provide data or information on a particular location is only possible if there is robust synchronicity (SYN). Furthermore, modality-selection (MDS) can amplify the degree of de-individuation and co-presence (DCP). Whether it is a phone call, texting, video conferencing or some other mode of communication, synchronicity (SYN) can certainly heighten and lessen the impact of de-individuation and co-presence (DCP).

These communication processes and capabilities are closely intertwined, resulting in dynamic interactions and reciprocal influences. Collectively, they cause an increase in mobile communication fluidity and promptness (figure 1). Specifically, they allow a person to frequently engage in multiple conversations or data sharing, and also enjoy immediacy, spontaneity, and gratification. These in turn lead to more frequent and more productive communicative activities.

\section{Research issues}

The first and foremost part of our agenda ought to be the validation of the presented media capabilities and characteristics. Each of these capabilities must be validated under various different contexts and using different variables. 


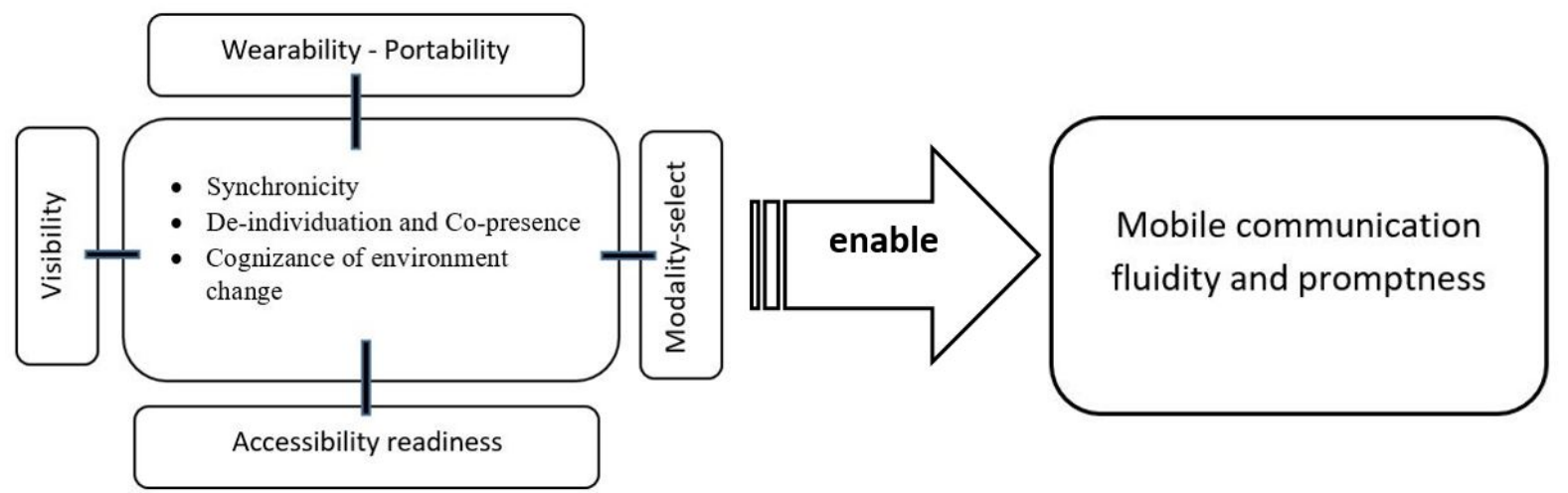

Figure 1 Media capabilities illustration

Table 1 Construct Summary

\begin{tabular}{|c|c|c|}
\hline Constructs & Abbrev. & Definitions \\
\hline \multicolumn{3}{|l|}{ Communication processes } \\
\hline Synchronicity & SYN & $\begin{array}{l}\text { Flawless and simultaneous communication between two or more } \\
\text { parties. }\end{array}$ \\
\hline De-individuation and Co-presence & DCP & $\begin{array}{l}\text { When a person simply observes an on-going group communication } \\
\text { without taking a part in that communication (de-individuation), which } \\
\text { in turn may allow the person to be involved in more than one online } \\
\text { rendezvous (co-presence) }\end{array}$ \\
\hline Cognizance of environment change & CEC & Changing locations and its influence on communication \\
\hline \multicolumn{3}{|l|}{ Communication capabilities } \\
\hline Modality-select & MDS & $\begin{array}{l}\text { A person's freedom to choose or switch back and forth from one } \\
\text { communication mode to another. }\end{array}$ \\
\hline Accessibility readiness & ARD & The level of readiness of a mobile technology for communication. \\
\hline Wearability & WRB & $\begin{array}{l}\text { How well an item of mobile technology conforms to a person's physical } \\
\text { needs }\end{array}$ \\
\hline Visibility & VSB & $\begin{array}{l}\text { The degree to which a piece of mobile technology's outside appearance } \\
\text { draws the attention of other people. }\end{array}$ \\
\hline
\end{tabular}

Table 2 Comparison between technologies

\begin{tabular}{|c|c|c|c|c|c|c|c|}
\hline $\begin{array}{c}\text { Social media integrated } \\
\text { with (device below) }\end{array}$ & SYN & DCP & CEC & MDS & ARD & WRB & VSB \\
\hline Personal computer & high & high & N/A & low & low & N/A & N/A \\
\hline Smartphone & high & high & high & high & med & med & high \\
\hline Google Glass & low-med & low-med & high & low-med & high & high & med \\
\hline Apple Watch & low-med & low-med & high & low-med & high & high & low \\
\hline
\end{tabular}


In computer-mediated communication (CMC), there have been a slew of various contexts and different variables by which such capabilities were tested-task type, group size, member dispersion, member familiarity, member attributes, member interaction, meeting or communication duration, anonymous identity, gender, meeting frequency, social influence, relevant background, member conflicts and more [1], [11], [14], [30]. In a similar manner, mobile-mediated communication (MMC) needs to be tested for each capabilities' legitimacy and efficacy. It is reasonable to expect that the capabilities will generate different processes and results than those found in CMC. Therefore, the focus should be on defining the user perceptions and attitudes that may trigger certain MMC-related behaviors.

Secondly, researchers must keep in mind that MMC fosters an environment that invites value creation. For example, Sørensen [29] discusses the value creationenabling capabilities of technology, such as computers as medium [2]. Specifically focusing on work environment, his study introduced the notion of enterprise mobility services. Given the capabilities our theory examines, there can now be a number of progressive services and products at the enterprise level. For instance, a few decades ago, the notion of virtual company based on e-commerce was simply a dream. But currently, we are looking at enterprise mobility that is primed and able.

Thirdly, it is important that future research considers human factors and behaviors. First, the modality-selection attribute (MDS) introduces a new area that has not been investigated by any previous IS studies. Furthermore, certain places or situations recommend one mode over the others, such as our preference for texting rather than making a voice call when in a classroom, church or on public transit.

From these considerations, the following questions arise: What factors influence a person's decision to choose and use a certain media mode? What are user's the motivations or rationales? How does this decisionmaking differ between a new call and returning call? How significant are the other relevant factors, such as task type and urgency, in affecting this modal selection? Are there any other major influencing factors such as societal, age, occupational, or cultural? How is "efficiency" defined in this realm? These and other similar questions must be worked on.

Fourth, the wearability-portability (WRB) and visibility (VSB) capabilities require more serious exploration. The concept of ubiquity or "anywhere and anytime" communication has been present in and has been used in many previous technological studies. But WRB and VSB take this concept to a new level. Being able to wear technology on one's face (Google Glass) or on the wrist (Apple Watch) is a different ubiquitous experience than being able to use an Internet-connected personal computer at any location. The cognitive interplay between a person, device, and communication process is different in these two CMC scenarios.

Lastly, but certainly not the least, a set of measuring instruments are needed for each of these constructs. There must be a set of validated and empirically-tested measuring instruments if we are to to examine a mobile technology according to this theoretical framework. Each instrument must be objective and testable in order to support this framework.

\section{References}

[1] M. Alavi, "Computer-mediated collaborative learning: An empirical evaluation,” MIS Quarterly, 18(2), June 1994, pp. 159-174.

[2] Andersen, P.B., B. Holmqvist and J.F. Jensen, (eds) The computer as medium. Cambridge University Press. 1993

[3] A. Barr, "Google Glass Loses Focus of Some Developers," The Wall Street Journal, Printed on November 14,2014

[4] M. Beigl, H.W. Gellersen and A. Schmidt, "Mediacups: experience with design and use of computer-augmented everyday artefacts," Computer Networks, 35(4), 2001, pp. 401-409.

[5] C. Bettini, S. Jajodia, P. Samarati, and S.X. Wang. "Privacy in location-based applications: research issues and emerging trends", Springer Science \& Business Media, Vol. $5599,2009$.

[6] Castells, M., M. Fernández-Ardèvol, and J.L. Qiu, Mobile Communication and Society: A Global Perspective. Cambridge, MA, U.S.A.: MIT Press, 2009.

[7] K. Choi and I. Im, "A Comparative Group Collaborative Communication Analysis of Mobile Microblogging and Nonmobile Online Message Board," International Journal of Electronic Commerce, 19(4), Summer 2015, pp. 1-24.

[8] R.L. Daft and R.H. Lengel, "Organizational information requirements, media richness and structural design," Management science, 32(5), 1986, pp. 554-571.

[9] L. Davidson, "Want a response from your teen? Text, don't call," Printed on Jul. 29, 2013,

http://www.today.com/parents/want-response-your-teen-textdont-call-6C10761156

[10] A.R. Dennis, R.M. Fuller, and J.S. Valacich, "Media, tasks, and communication processes: A theory of media synchronicity," MIS quarterly, 32(3), 2008, pp. 575-600. 
[11] G. DeSanctis and R.B. Gallupe, "A foundation for the study of group decision support systems," Management Science, 33(5), 1987, pp. 589-609

[12] Facebook.com, Data retrieved May 22, 2015 http://newsroom.fb.com/company-info/

[13] L. Festinger, A. Pepitone, \& T. Newcomb, "Some consequences of de-individuation in a group," the Journal of Abnormal and Social Psychology, 47(2S), 382. 1952.

[14] J. Fjermestad and S.R. Hiltz, "An assessment of group support systems experimental research: methodology and results,” Journal of Management Information Systems, 15(3), 1998, pp. 7-149.

[15] G. A. Fowler, “One-Minute Review: Amazon's 'Flow' Image Recognition Beats Barcode Scans," The Wall Street Journal, Printed on February 5, 2014

[16] Gardner research, "Market Share: Devices, All Countries, 4Q14 Update." Date retrieved May 12th 2015, URL http://www.gartner.com/document/2985017.

[17] V. Goel, "Facebook's Growth Slows Slightly, but Mobile Shift Intensifies," The New York Times, Printed on April 22, 2015.

[18] A. Goldfarb, "The Internet Killed Distance. Mobile Computing Brought It Back," MIT Technology Review, Data retrieved May 31, 2015,

http://www.technologyreview.com/news/520796/theinternet-killed-distance-mobile-computing-brought-it-back/

[19] C. Gummer, "Zalando Testing Mobile Clothing Recognition App," The Wall Street Journal, Printed on August 29, 2014.

[20] R. Haines and C. Mann, "A new perspective on deindividuation via computer-mediated communication," European Journal of Information Systems, March 2011, 20(2), pp 156-167, 2011.

[21] A. Hesseldahl, Printed on Apr 11, 2016, "After Worst Year Ever, PC Sales Off to Another Bad Start in 2016," Retrieved from

http://www.recode.net/2016/4/11/11586044/after-worst-yearever-pc-sales-off-to-another-bad-start-in-2016
[22] IDC, International Data Corporation (IDC) Worldwide Quarterly Mobile Phone Tracker, Date retrieved May 11, 2015. URL http://www.idc.com/prodserv/smartphonemarket-share.jsp

[23] A.Lenhart, R. Ling, S. Campbell and K. Purcell, "Teens and Mobile Phones," Pew Research Center, Printed on April 20, 2010, http://www.pewinternet.org/2010/04/20/teens-andmobile-phones/

[24] J. Macoubrie, "Public perceptions about nanotechnology: Risks, benefits and trust," Journal of Nanoparticle Research, 6(4), 2014, pp.395-405.

[25] F. Manjoo, "For Verizon and AOL, Mobile Is a Magic Word," New York Times, printed on May 13, 2015.

[26] J. Marshall, "Foursquare Launches New Location-Based Ad Offering," The Wall Street Journal, Printed on April 14, 2015

[27] R. McMillan, Printed on Jan. 12, 2016, "PC Sales Drop to Historic Lows" Retrieved from ttp://www.wsj.com/articles/pc-sales-drop-to-historic-lows1452634605

[28] T.P. Moran and P. Dourish, "Introduction to this special issue on context-aware computing," Human-Computer Interaction, 16(2-4), 2001, pp.87-95.

[29] Sørensen, C., Enterprise Mobility: Tiny Technology with Global Impact on Work, Palgrave Macmillan, first edition, November 15, 2011.

[30] S. Strauss and J.E. McGrath, "Does the medium matter? The interaction of task type and technology on group performance and member reactions." Journal of Applied Psychology, 79(1), 1994, pp. 87-97.

[31] Twitter.com, Date retrieved May 22, 2015 https://about.twitter.com/company

[32] A. Wigfield and J.S. Eccles, "Expectancy-value theory of achievement motivation," Contemporary educational psychology, 25(1), 68-81. 2000.

[33] R. Winkler, "Google's Spero on Mobile Commerce: "The Consumer Has All the Power," The Wall Street Journal, Printed on November 14, 2014. 